Research Paper

\title{
PD-LI Expression in Clear Cell Renal Cell Carcinoma: An Analysis of Nephrectomy and Sites of Metastases
}

\author{
L.B. Jilaveanu', B. Shuch ${ }^{2}$, C. R. Zito ${ }^{1,3}$, F. Parisi ${ }^{4}$, M. Barrr, Y. Kluger ${ }^{4}$, L. Chen ${ }^{5}$ and H. M. Kluger ${ }^{1}$ \\ 1. Departments of Medicine, Yale University School of Medicine, New Haven, CT, USA \\ 2. Department of Urology, Yale University School of Medicine, New Haven, CT, USA \\ 3. Department of Biology, School of Health and Natural Sciences, University of Saint Joseph, West Hartford, CT, USA \\ 4. Department of Pathology, Yale University School of Medicine, New Haven, CT, USA \\ 5. Department of Immunobiology, Yale University School of Medicine, New Haven, CT, USA \\ $\triangle$ Corresponding author: Dr. Brian Shuch, Assistant Professor. Department of Urology, Yale School of Medicine, 333 Cedar St. New Haven, \\ CT 06520. Tel: 203-737-6332 Fax: 203-785-6475; Email: brian.shuch@yale.edu
}

(C) Ivyspring International Publisher. This is an open-access article distributed under the terms of the Creative Commons License (http://creativecommons.org/ licenses/by-nc-nd/3.0/). Reproduction is permitted for personal, noncommercial use, provided that the article is in whole, unmodified, and properly cited.

Received: 2013.11.19; Accepted: 2013.12.22; Published: 2014.01.24

\begin{abstract}
Background: Expression of programmed death ligand (PD-LI/B7-HI/CD274) represents a mechanism of immune escape for renal cell carcinoma (RCC) cells. Drugs blocking PD-LI or its receptor are in clinical development and early data suggests that tumor PD-LI expression may predict response.

Patients and Methods: A tissue microarray (TMA) consisting of four biopsy cores from 34 matched pairs of nephrectomy and metastatic sites of clear cell RCC was used to assess PD-LI expression by quantitative immunofluorescence. Assessment of intra- and inter-tumor heterogeneity and primary and metastatic tumor expression was performed using a method of Automated Quantitative Analysis (AQUA).

Results: The median AQUA scores were higher in metastatic than primary specimens $(P<$ 0.000 I). The correlation between PD-LI expression in matched primary and metastatic specimens was weak $(R=0.24)$. Within a given tumor, variable PD-LI staining heterogeneity was seen, however the degree of heterogeneity was similar in primary and metastatic sites $(P=0.482)$.

Conclusions: The weak correlation between PD-LI expression in primary and metastatic sites for a given patient suggests that expression in nephrectomy specimens cannot be used to select metastatic RCC patients for PD-LI and PD-I inhibitors. The intra-tumor heterogeneity seen in both primary and metastatic specimens indicates that a single core biopsy might not be sufficient to determine PD-LI expression.
\end{abstract}

Key words: PD-L1, renal cell carcinoma

\section{Introduction}

Renal cell carcinoma (RCC) has long been recognized as an immunoresponsive tumor with spontaneous regressions occurring on rare occasions.[1] This led investigators to focus on immunomodulatory strategies to stimulate anti-tumor activity. As RCC appeared to be a chemoresistant tumor, there was excitement when DeKernion and colleagues noted clinical activity with Interferon-a.[2] Later, Rosenberg and colleagues reported the results of treatment with high dose Interleukin-2 (IL-2) in patients with RCC.[3] While toxicity can be significant with IL-2, complete responses were observed in a small percentage of patients. $[4,5]$ While complete responses are uncommon, those that occur are typically durable.[6] Based on these responses, in 1992 the Food and Drug Administration (FDA) approved IL-2 for the use in metastatic RCC.

With the discovery of VHL alterations in the 
majority of clear cell RCCs, targeted therapies were studied in this population.[7] Loss of VHL leads to dyregulated hypoxia inducible pathways and highly vascular renal tumors.[8] Therapies targeting angiogenesis were introduced in early 2003 with trials of inhibitors of VEGF-R2 and its ligand, VEGF, as well as the downstream mediator, mTOR. $[9,10]$ Since that time, seven agents targeting the angiogenesis pathway have been FDA approved for use in patients with advanced kidney cancer. While the response rates appear higher than historic series with IL-2, complete responses are exceedingly rare, and when they occur, they are not durable.[11] As immunotherapy is the only form of therapy known to produce durable responses, it remains a viable option for select patients.

With an improved understanding of how tumor cells evade anti-tumor response, there has been considerable effort to exploit these mechanisms for therapeutic targeting. While cytotoxic lymphocytes are known to produce anti-tumor activity, $\mathrm{T}$ cell response can be modulated by activating and inhibitory co-receptors. Understanding how these secondary signals modulate $\mathrm{T}$ cell response has led to new therapeutic strategies. CLTA-4 is an inducible T cell receptor that when triggered can inhibit T-cell proliferation/function. Preclinical models demonstrated that CTLA-4 blockade could augment anti-tumor response[12]. A humanized monoclonal antibody, now approved for use in metastatic melanoma, showed activity in a phase II trial with RCC.[13] Another mechanism of immune evasion is tumor expression of programmed death ligand 1 (PD-L1) (also known as B7-H1 or CD274) on many cancer types including RCC. Binding of PD-L1 to the co-stimulatory receptor on T cells, PD-1, promotes inactivation and apoptosis of activated anti-tumor T cells.[14] In RCC, Thompson et al demonstrated that two-thirds of clear cell RCC had PD-L1 expression and those with high expression had worse cancer-specific survival.[15, 16] The same group also investigated tumor-infiltrating lymphocytes (TIL) in primary RCC and demonstrated that TILs also express PD-L1 and that high expression on either tumors or TILs was associated with advanced stage, worse prognosis and rapid metastatic progression. $[15,16]$ Further studies have shown that blockade of the PD1-PD-L1 pathway augments anti-tumor activity provides therapy for multiple solid tumors, including RCC.[17]

PD-1 and PD-L1 inhibiting drugs are currently in clinical development. The PD-1 inhibitor BMS-936558 (Nivolumab) has dramatic activity with little toxicity in RCC, and early data suggest that PD-L1 tumor expression might be necessary for response.[18] The PD-L1 inhibitor MPDL-3280A (Genentech) has also shown activity in RCC, and
PD-L1 expression in the tumor micro-environment might be needed for drug activity.[19,20]

As additional studies are being conducted on the predictive value PD-L1 in pretreatment specimens, our purpose was to establish patterns of PD-L1 expression in both primary and metastatic specimens and to determine whether this expression is homogenous or not. Previous immunohistochemical analysis of both fresh-frozen and/or paraffin embedded tissues showed that PD-L1 was variably expressed by primary and metastatic RCC tumor cells as well as TILs and although Thompson et al report slightly higher rates of PD-L1 expression in metastatic versus primary specimens, a thorough comparison in matched samples has not been made.[15, 16, 21] As tissue from primary tumors and distant sites may be assessable for IHC, we investigated the PD-L1 expression in both tissue types, utilizing a specially constructed tissue microarray containing matched tumor pairs from the same patient, incorporating four specimen cores from each tumor.

\section{Materials and Methods}

\section{Tissue microarray (TMA) construction}

TMAs were constructed from a cohort of matched primary and metastatic RCC tumors of thirty-four patients, who had undergone resections between 1972 and 2011. Collection of specimens and clinical data was conducted with approval of a Yale University Institutional Review Board. Each tumor site was represented by four cores from different areas of the specimen; two cores from each tumor site were included in each of two TMA blocks (8 cores of each patient in total). Specimen accrual, tumor characteristics (including size, grade, histology and site of metastases), and other clinical information have been described.[22, 23] Briefly, this cohort included 20 males $(60 \%)$ and 14 females (40\%). Median age at diagnosis was 56 years of age (range 17 to 72 ). A total of 31 RCC patients had pure clear cell carcinoma (91\%) and 3 had mixed sarcomatoid and clear cell histology $(9 \%)$. The majority of metastatic specimens were lung metastases but also included several other visceral sites, skin, soft tissue and soft tissue components of bone metastases. Both TMA blocks contained identical cell lines, cored from cell pellets, as previously detailed for normalization between TMA blocks.[22, 23] To verify antibody specificity, we used small TMAs containing placental and tonsil tissue (known to be positive for PD-L1 expression) and pellets from MEL-624 cell lines, overexpressing or not overexpressing PD-L1, as previously described.[14] 


\section{Immunofluorescence and Automated Quantitative Analysis (AQUA)}

For AQUA analysis, 2 pairs of slides (containing two cores from different areas of each matching primary and metastatic tumor per patient) or test arrays of cores from FFPE placental tissue and MEL624 cells not overexpressing or overexpressing PD-L1 were concomitantly stained. TMAs were heated in an oven for 30 minutes at $60^{\circ} \mathrm{C}$, deparaffinized and rehydrated through xylenes and serial dilutions of ETOH and $\mathrm{H}_{2} \mathrm{O}$. Slides were incubated in antigen retrieval buffer (DAKO, Carpinteria, CA,) supplemented with $0.3 \%$ Tween 20 and boiled at $100^{\circ} \mathrm{C}$ for 10 minutes. Slides were then incubated in peroxidase blocking reagent (ready to use reagent, K1500, DAKO) for $5 \mathrm{~min}$ at room temperature. To block unspecific staining, slides were incubated at room temperature for $15 \mathrm{~min}$ in ACE blocking buffer. To block endogenous biotin, slides were first incubated in Avidin blocking solution followed by Biotin blocking reagent (Vector Laboratories, Burlingame, CA) for 15 minutes each at $37^{\circ} \mathrm{C}$. One pair of slides was incubated with the primary antibody (anti PD-L1 monoclonal mouse antibody, clone $5 \mathrm{H} 1$, generated by Dr. Lieping Chen, Yale University, New Haven, CT) diluted at 1:75 in ACE block at $4^{\circ} \mathrm{C}$ overnight.[14] This antibody was previously produced through immunization of BALB/C mice with human PD-L1 Ig and has been validated in previous publications.[14, 15] As a negative control, a second pair of TMA slides was incubated overnight in ACE block only. All TMAs were then incubated for 45 minutes at room temperature with biotynilated anti-mouse IgG (1:500, BA-2001, Vector laboratories), followed by the ABC kit (PK-6100,Vector Laboratories) for 15 minutes incubation at room temperature. Slides were covered with amplification reagent (K1500, DAKO) for 4 minutes at room temperature followed by Streptavidin-HRP (K1500, DAKO) for 15 minutes at room temperature and then Cyanine 5-tyramide (Perkin Elmer, Waltham, MA, USA) for target detection. Slides were incubated twice for 7 minutes with $100 \mathrm{mM}$ benzoic hydrazide (Sigma, St. Louis, MO) and 50mM hydrogen peroxide in PBS to quench the HRP. A tumor mask was created by incubation with rabbit anti-cytokeratin (Dako, dilution 1:100) and CAIX (Novus Biologicals, Littleton, CO, dilution 1:2000) for 2 hours at room temperature. Goat anti-rabbit HRP-decorated polymer backbone (Envision, Dako) was used as a secondary reagent. Incubation with Cyanine 2-tyramide (Perkin Elmer) in the supplied amplification buffer was used to visualize tumor mask. A nuclear mask was created by incu- bating the slides with 4, 6-diamidine-2-phenylindole (DAPI) (Invitrogen, Carlsbad, CA, dilution 1:500). Coverslips were mounted with ProLong Gold anti-fade reagent with DAPI (Invitrogen).

\section{Automated Image Acquisition and Analysis}

Images were acquired and analyzed as described.[24] Monochromatic, high-resolution (1024 × 1024 pixel) images were obtained of each histospot. Tumor mask was created from the Cy2 signal and DAPI was used to identify the nuclei. PD-L1 signal was visualized by Cy5, compartmentalized and expressed as the average signal intensity within the assayed component (AQUA score), with scores on a scale of 0-255.

\section{Data Analysis}

JMP version 5.0 software was used for analysis (SAS Institute, Cary, NC). Associations between continuous AQUA scores and clinical/pathological parameters were assessed by analysis of variance. Correlations between the AQUA scores of matched primary and metastatic histospots were calculated by the log rank method.

\section{Results}

In this study we utilized a mouse monoclonal anti-PD-L1 antibody (5H1 clone) which was previously produced through immunization of BALB/c mice with human PD-L1 Ig. This antibody was extensively tested and validated in previous publications. $[14,15]$ To confirm the PD-L1 antibody's specificity with immunofluorescence, we measured levels of PD-L1 expression on arrays that contain positive tissue controls (human placenta and tonsil and MEL624 cell line pellets overexpressing PD-L1). As seen in Figure 1, the antibody was highly specific for its target and showed strong immunoreactivity with the MEL624 ${ }^{\text {PD-L1+ }}$ cells, but not the parental MEL624 cells.

The RCC TMA, containing eight cores total from each patient (four cores from primary tumors and four from metastatic) was stained to evaluate PD-L1 expression and to investigate inter- and intra-tumor heterogeneity. PD-L1 expression in the tumor mask was quantified. Staining was membranous/cytoplasmic. Tumor spots were excluded from the analysis if they had abundant necrotic tissue, insufficient tumor cells, loss of tissue in the spot during immunostaining, or showed high background on the negative control slide (incubated with secondary antibody but not with anti-B7H1 antibody) due to high endogenous biotin. 

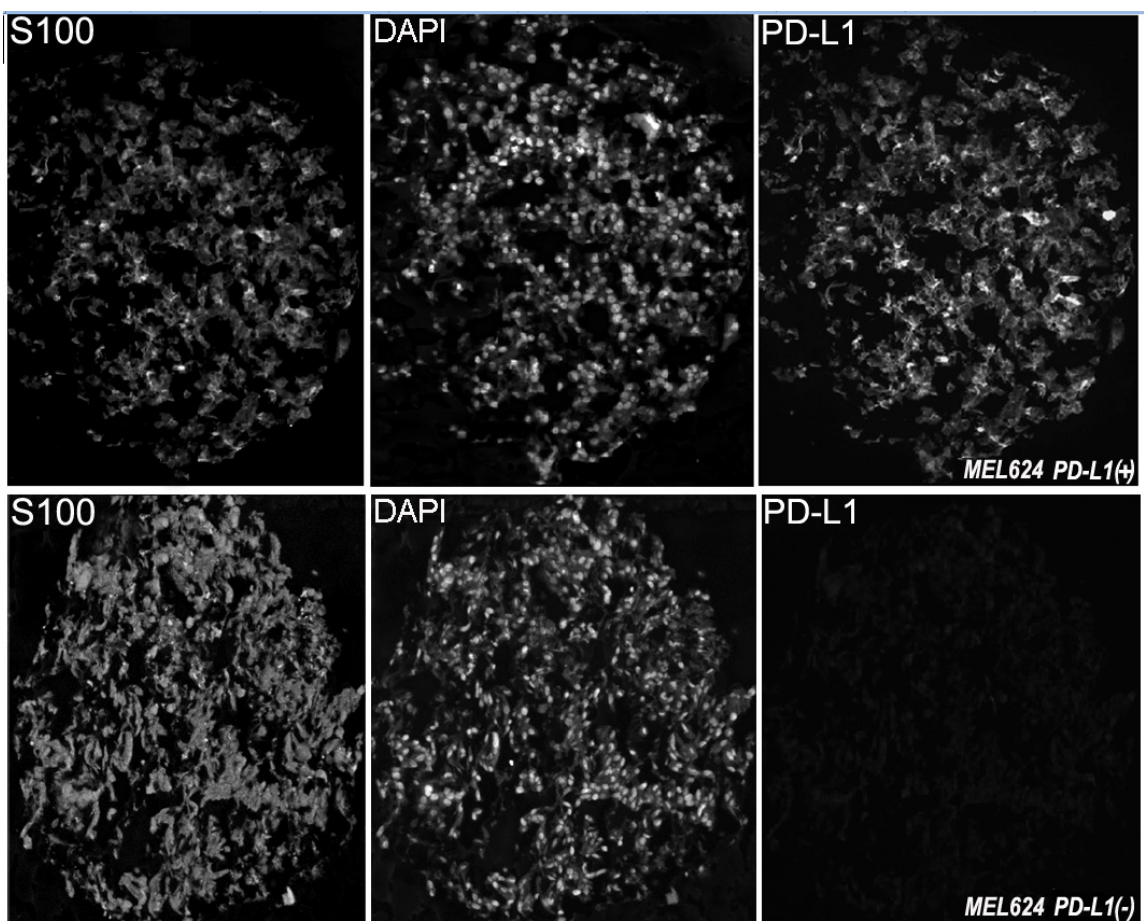

Figure I. Examples of PD-LI fluorescent staining, shown at $\times 10$ magnification, in two melanoma histospots of formalin fixed paraffin embedded MEL624 cells transfected to overexpress PD-LI or non-transfected. Anti-SI00 conjugated to Cy2 was used to visualize the cytoplasm while DAPI (4,6-diamidine-2-phenylindole) was used to identify nuclei. Cy5 was used to visualize the target.

AQUA scores ranged from 5.1 to 32.7 (mean: 15.5; median: 15.5) for primary RCC tissue, and from 8.1 to 51.7 for metastatic tissue (mean: 21.9; median: 21.7). Patients often have available tissue blocks from nephrectomy specimens, but not from metastatic sites for analysis of biomarker expression, yet metastatic specimens are typically treated with systemic therapy, after nephrectomy. To determine whether expression of PD-L1 in nephrectomy specimens can be used as a surrogate for expression in the sites of metastases (and vice versa), we examined the correlation of marker expression (mean AQUA scores for all four cores) between the matched specimens using the Pearson correlation test. A weak association was found between PD-L1 expression in matched primary and metastatic specimens $(\mathrm{R}=0.24)$.

Seeing that systemic therapy is most often given to treat metastatic disease, we next assessed the difference in PD-L1 expression between primary and metastatic tissues by paired $t$ tests. Expression was significantly higher in metastatic versus primary tissue cores $(P<0.05)$, as shown in Figure 2.

A number of prior reports have suggested that PD-L1 expression within a tumor might be heterogeneous.[25] Seeing that in metastatic patients the only available tumor is often core biopsies rather than whole tissue blocks, we next attempted to determine whether the degree of heterogeneity varies between primary and metastatic sites, using the TMA cores as surrogates for core biopsy specimens. To further quantify the intra-tumor heterogeneity of PD-L1 expression within the primary and matched metastatic tumors utilizing the four different expression measurements for each tumor block, a composite median absolute deviation (MAD) score was generated for each block and patient, as described previously.[22] The Wilcoxon paired, two-sided signed rank test, showed that overall the heterogeneity between primary and metastatic tumors did not significantly differ $(P=0.482)$ (Figure 3$)$. Figure 4 shows an example of heterogeneous PD-L1 staining of four corresponding primary tumor cores from a patient whose AQUA scores for the four cores ranged from 12.2 to 37.8.

\section{Discussion}

Despite a possible improvement in median survival of RCC patients treated with VEGF or VEGF-R targeting agents, long-term survivors ( $>5$ years) are rare.[26] Due to the lack of complete responses with targeted therapy, there has been resurgent interest in immunotherapy.[27] Besides BMS-936558 (Nivolumab), another humanized anti-PD1 antibody, MK-3475 (Lambrolizumab), has shown activity and acceptable toxicity in a phase I trial with metastatic melanoma.[28] Other similar strategies targeting PD-L1 are in development and include antibodies BMS-936559 and MPDL3280A, and these newer agents are being studied in RCC.[29] 


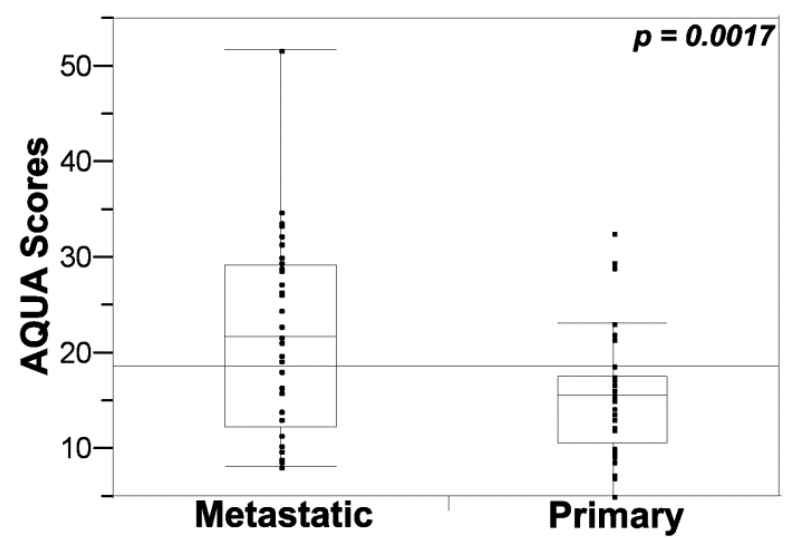

Figure 2. PD-LI expression (tumor mask AQUA scores) in metastatic and primary specimens. Unpaired t tests showed that PD-LI expression was significantly higher in metastatic versus primary tissue cores.
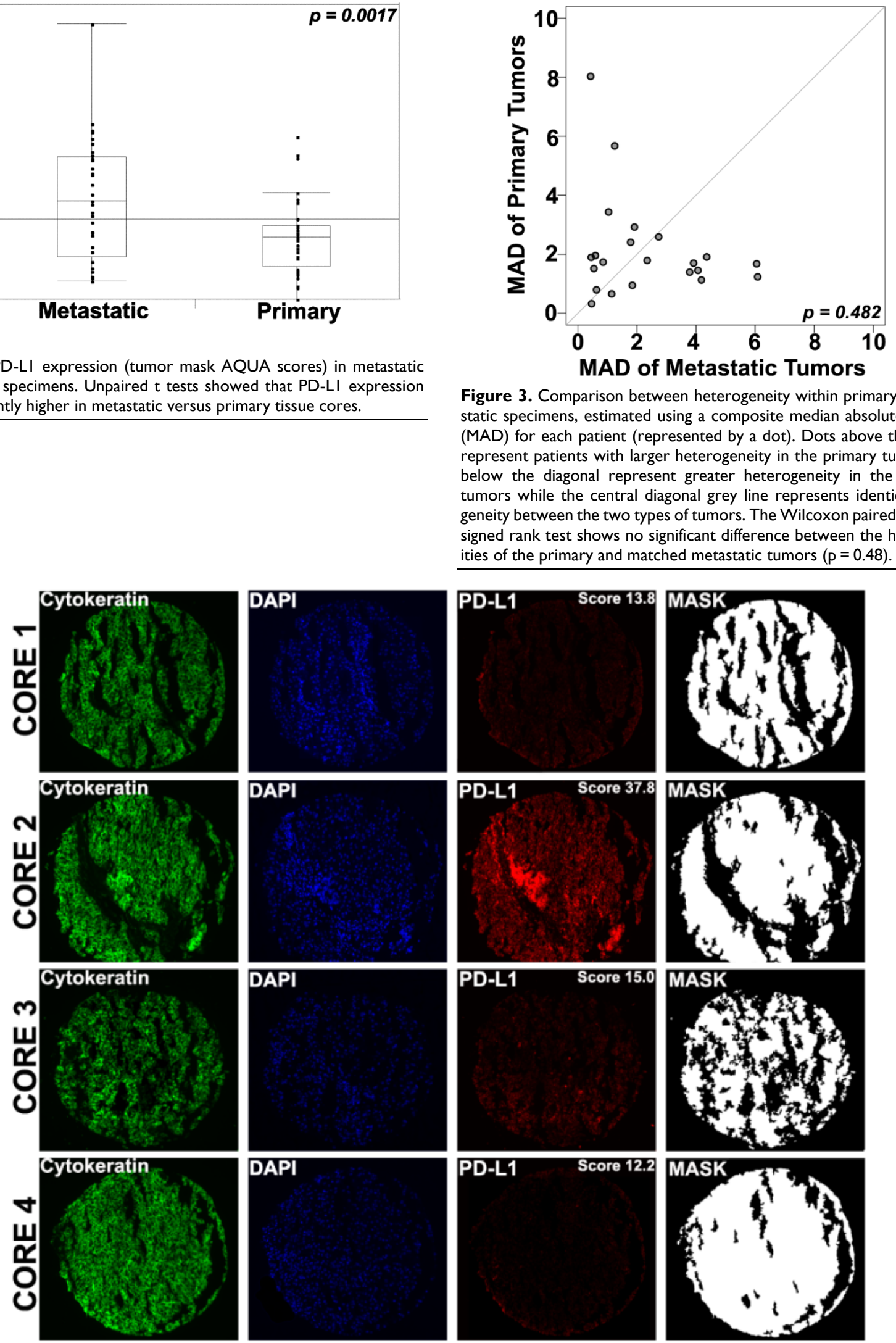

Figure 3. Comparison between heterogeneity within primary and metastatic specimens, estimated using a composite median absolute deviation (MAD) for each patient (represented by a dot). Dots above the diagonal represent patients with larger heterogeneity in the primary tumors; dots below the diagonal represent greater heterogeneity in the metastatic tumors while the central diagonal grey line represents identical heterogeneity between the two types of tumors. The Wilcoxon paired, two-sided signed rank test shows no significant difference between the heterogeneities of the primary and matched metastatic tumors $(p=0.48)$.
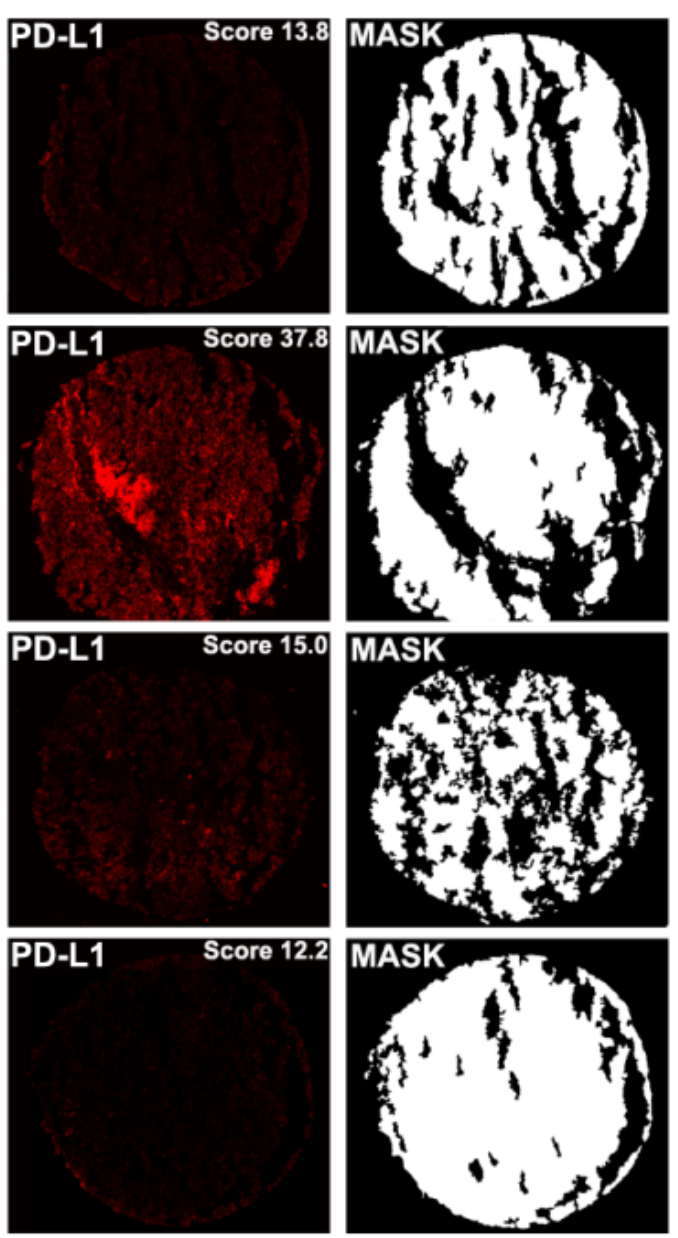

Figure 4. Examples of PD-LI fluorescent staining, shown at $\times 10$ magnification in four distinct cores from different areas of a primary tumor corresponding to a single patient. AQUA uses cytokeratin to define the tumor mask, DAPI to define the nuclear compartment, and Cy5 for the target (PD-LI). The cytoplasmic compartment is generated by subtracting the nuclear compartment from the cytokeratin mask. PD-LI expression is then measured within each compartment within the tumor mask, and each spot is assigned a score based on pixel intensity per unit area. PD-LI expression (measured in the tumor mask compartment) in cores I to 4 corresponds to AQUA scores of I3.8, 37.8, I5.0 and I2.2 respectively. 
PD-L1 and PD-1 inhibiting drugs exhibit a favorable toxicity profile, but activity is limited to a subset of patients, and there is therefore great need to develop predictors of response to these therapies to facilitate patient selection. In RCC several immunohistochemical predictive biomarkers have been proposed for clear cell RCC including pS6 for temisorimus therapy and CAIX for high dose IL-2.[30, 31] In developing candidate predictive biomarkers, several issues will make validation and adoption challenging in RCC since we may not know which tissue type (primary or metastatic tissue) to test and how many biopsy cores are needed. Unlike other solid tumors, RCC is unique in that debulking nephrectomy has been the mainstay of therapy even in the setting of metastatic disease. However, access to tumor from distant disease sites may be feasible, raising the question of which tissue to study, the primary which is resected or the distant sites which will need the benefit of systemic therapy. While metastatic sites may have originated from the primary tumor, several studies have demonstrated a different immunohistochemical or mutation profile compared to the primary.[30, 32, 33] This significant degree of genetic heterogeneity may present a major challenge to biomarker development.[33]

As PD-1 and PD-L1 targeting therapy continues in clinical development, our purpose was to better characterize PD-L1 tissue expression. Our study has several important findings that have implications for biomarker development for this class of drugs. First, while the primary renal tumor and distant sites can have biologic similarities,[34] we observed only a weak association in PD-L1 expression between the primary tumor and distant metastases $(R=0.24)$. As distant sites of disease may represent aggressive subclones that disseminated from the primary tumor, it is plausible that these clones involved additional genetic derangements that allowed their systemic dissemination. Not surprisingly, the PD-L1 expression was significantly higher in metastatic sites compared to the nephrectomy cores $(P<0.05)$. Data from Thompson and colleagues suggested this finding when they evaluated 196 primary renal tumors and 26 metastases. In their analyses, higher expression was more common in the distant sites, and this was confirmed by our studies, but unlike our series, that series included only one patient with both tissue types available.[16] Second, we investigated the degree of tumor heterogeneity, which has been shown to be quite significant in primary kidney cancer[33]. In our study, variable heterogeneity was found in different patients; some had homogenous tumors, while others did not, suggesting that in some patients a single core might represent PD-L1 expression of an entire tumor, while in others it might not. The degree of heterogeneity did not differ between primary and metastatic sites, indicating that more than one core biopsy, or even an entire tumor block might be needed to determine PD-L1 expression in a tumor. As clinical trials continue to investigate anti-PD-L1 therapy and the predictive value of PD-L1 tumor expression, this degree of tissue heterogeneity must be taken into account.

The strengths of this study include our unique tissue microarray design, containing matched primary and metastatic tissue from the same patient. While some microarrays contain distant sites, these often are not available from the same patient. Additionally while immunohistochemistry is often criticized due to the subjective and qualitative nature, we utilized an automated method. Prior studies have evaluated PD-L1 expression in RCC, but our use of the AQUA system allowed an objective and quantitative assessment of tumor staining. One limitation of our study is the use of metastatic samples from various organ sites, potentially influencing expression via differences from the organ-specific tumor microenvironment.

TMA cores are useful surrogates for core biopsies, which are typically obtained to access tissue from metastatic sites in patients with unresectable disease. Camp and colleagues evaluated the correlation of TMAs to whole tissue sections and concluded that 2 separate cores allowed accurate assessment of protein expression, although this is clearly biomarker dependent, as heterogeneity varies from marker to marker[35]. Other studies have looked at PD-L1 expression as a prognostic variable and in the context of response to systemic therapy and used a cut point of percentage positivity of $\geq 5 \%$. [15, 16, 18, 20,36] These studies have not taken into consideration intensity of staining, as done with our method, which utilizes both intensity and area of positive expression. In our study we did not used a predefined cut point of positive versus negative expression, as this will be determined in the context of response to anti-PD-L1 drugs.

\section{Conclusions}

Early phase trials with anti-PD-L1 and anti-PD-1 antibodies have shown clinical activity in RCC and early biomarker assessment suggests PD-L1 tumor expression may influence response. We demonstrate that distant sites have greater PD-L1 expression than the primary tumors. Additionally there is only weak correlation in staining between the matched primary and distant metastasis, suggesting that the primary tumor is not an adequate surrogate for determining PD-L1 expression in metastatic sites. Tissue heterogeneity in PD-L1 expression in both primary and 
metastatic sites indicates that a single core biopsy might not be sufficient to determine tumor PD-L1 expression. A cut-point for positivity has to be determined in the context of response to this class of therapies.

\section{Acknowledgements}

This publication was made possible by CTSA Grant Number KL2 TR000140 from the National Center for Advancing Translational Science (NCATS), a component of the National Institutes of Health (NIH) (to L. Jilaveanu). Its contents are solely the responsibility of the authors and do not necessarily represent the official view of NIH. This work was also supported in part by NIH grants including the K24CA172123 (to H. Kluger) and R0-1 CA158167 (to H. Kluger), and the American Cancer Society Award M130572 (to H. Kluger).

We thank Mr. Joshua Sznol for technical input.

\section{Competing Interests}

The authors have declared that no competing interest exists.

\section{References}

1. Freed SZ, Halperin JP, Gordon M. Idiopathic regression of metastases from renal cell carcinoma. J Urol. 1977; 118: 538-42.

2. deKernion JB, Sarna G, Figlin R, Lindner A, Smith RB. The treatment of renal cell carcinoma with human leukocyte alpha-interferon. J Urol. 1983; 130: 1063-6.

3. Rosenberg SA, Lotze MT, Muul LM, Leitman S, Chang AE, Ettinghausen SE, et al. Observations on the systemic administration of autologous lymphokine-activated killer cells and recombinant interleukin-2 to patients with metastatic cancer. N Engl J Med. 1985; 313: 1485-92. doi:10.1056/NEJM198512053132327.

4. Rosenberg SA, Yang JC, Topalian SL, Schwartzentruber DJ, Weber JS, Parkinson DR, et al. Treatment of 283 consecutive patients with metastatic melanoma or renal cell cancer using high-dose bolus interleukin 2. JAMA. 1994; 271: 907-13.

5. Kammula US, White DE, Rosenberg SA. Trends in the safety of high dose bolus interleukin-2 administration in patients with metastatic cancer. Cancer. 1998; 83: 797-805.

6. Klapper JA, Downey SG, Smith FO, Yang JC, Hughes MS, Kammula US, et al. High-dose interleukin-2 for the treatment of metastatic renal cell carcinoma : a retrospective analysis of response and survival in patients treated in the surgery branch at the National Cancer Institute between 1986 and 2006. Cancer. 2008; 113: 293-301. doi:10.1002/cncr.23552.

7. Gnarra JR, Tory K, Weng Y, Schmidt L, Wei MH, Li H, et al. Mutations of the VHL tumour suppressor gene in renal carcinoma. Nat Genet. 1994; 7: 85-90.

8. Maxwell PH, Wiesener MS, Chang GW, Clifford SC, Vaux EC, Cockman ME, et al. The tumour suppressor protein VHL targets hypoxia-inducible factors for oxygen-dependent proteolysis. Nature. 1999; 399: 271-5.

9. Yang JC, Haworth L, Sherry RM, Hwu P, Schwartzentruber DJ, Topalian SL, et al. A randomized trial of bevacizumab, an anti-vascular endothelial growth factor antibody, for metastatic renal cancer. N Engl J Med. 2003; 349: 427-34. doi:10.1056/NEJMoa021491. 349/5/427 [pii].

10. Kuenen BC, Tabernero J, Baselga J, Cavalli F, Pfanner E, Conte PF, et al. Efficacy and toxicity of the angiogenesis inhibitor SU5416 as a single agent in patients with advanced renal cell carcinoma, melanoma, and soft tissue sarcoma. Clin Cancer Res. 2003; 9: 1648-55.

11. Albiges L, Oudard S, Negrier S, Caty A, Gravis G, Joly F, et al. Complete remission with tyrosine kinase inhibitors in renal cell carcinoma. Journal of clinical oncology : official journal of the American Society of Clinical Oncology. 2012; 30: 482-7. doi:10.1200/JCO.2011.37.2516.

12. Leach DR, Krummel MF, Allison JP. Enhancement of antitumor immunity by CTLA-4 blockade. Science. 1996; 271: 1734-6.

13. Yang JC, Hughes M, Kammula U, Royal R, Sherry RM, Topalian SL, et al. Ipilimumab (anti-CTLA4 antibody) causes regression of metastatic renal cell cancer associated with enteritis and hypophysitis. J Immunother. 2007; 30: 825-30. doi:10.1097/CJI.0b013e318156e47e.
14. Dong H, Strome SE, Salomao DR, Tamura H, Hirano F, Flies DB, et al. Tumor-associated B7-H1 promotes T-cell apoptosis: a potential mechanism of immune evasion. Nat Med. 2002; 8: 793-800. doi:10.1038/nm730.

15. Thompson RH, Gillett MD, Cheville JC, Lohse CM, Dong H, Webster WS, et al. Costimulatory B7-H1 in renal cell carcinoma patients: Indicator of tumor aggressiveness and potential therapeutic target. Proceedings of the National Academy of Sciences of the United States of America. 2004; 101: 17174-9. doi:0406351101 [pii]. 10.1073/pnas.0406351101.

16. Thompson RH, Gillett MD, Cheville JC, Lohse CM, Dong H, Webster WS, et al. Costimulatory molecule B7-H1 in primary and metastatic clear cell renal cell carcinoma. Cancer. 2005; 104: 2084-91. doi:10.1002/cncr.21470.

17. Iwai $Y$, Ishida M, Tanaka $Y$, Okazaki T, Honjo T, Minato N. Involvement of PD-L1 on tumor cells in the escape from host immune system and tumor immunotherapy by PD-L1 blockade. Proc Natl Acad Sci U S A. 2002; 99: 12293-7. doi:10.1073/pnas.192461099.

18. Topalian SL, Hodi FS, Brahmer JR, Gettinger SN, Smith DC, McDermott DF, et al. Safety, activity, and immune correlates of anti-PD-1 antibody in cancer. N Engl J Med. 2012; 366: 2443-54. doi:10.1056/NEJMoa1200690.

19. Herbst RS, Gordon MS, Fine GD, Sosman JA, Soria JC, Hamid O, et al. A study of MPDL3280A, an engineered PD-L1 antibody in patients with locally advanced or metastatic tumors. J Clin Oncol. 2013; 31(suppl): abstr3000.

20. Powderly JD, Koeppen H, Hodi FS, Sosman JA, Gettinger SN, Desai R, et al. Biomarkers and associations with the clinical activity of PD-L1 blockade in a MPDL3280A study. J Clin Oncol. 2013; 31(suppl): abstr3001.

21. Thompson RH, Kuntz SM, Leibovich BC, Dong H, Lohse CM, Webster WS, et al. Tumor B7-H1 is associated with poor prognosis in renal cell carcinoma patients with long-term follow-up. Cancer Res. 2006; 66: 3381-5. doi:66/7/3381 [pii]. 10.1158/0008-5472.CAN-05-4303.

22. Aziz SA, Sznol JA, Adeniran A, Parisi F, Kluger Y, Camp RL, et al. Expression of drug targets in primary and matched metastatic renal cell carcinoma tumors. BMC Clin Pathol. 2013; 13: 3. doi:10.1186/1472-6890-13-3.

23. Aziz SA, Sznol J, Adeniran A, Colberg JW, Camp RL, Kluger HM. Vascularity of primary and metastatic renal cell carcinoma specimens. J Transl Med. 2013; 11: 15. doi:10.1186/1479-5876-11-15.

24. Camp RL, Chung GG, Rimm DL. Automated subcellular localization and quantification of protein expression in tissue microarrays. Nat Med. 2002; 8: 1323-7. doi:10.1038/nm791.

25. Taube JM, Anders RA, Young GD, Xu H, Sharma R, McMiller TL, et al. Colocalization of inflammatory response with B7-h1 expression in human melanocytic lesions supports an adaptive resistance mechanism of immune escape. Sci Transl Med. 2012; 4: 127ra37. doi:4/127/127ra37 [pii] 10.1126 /scitranslmed.3003689.

26. Harshman LC, Xie W, Bjarnason GA, Knox JJ, MacKenzie M, Wood L, et al. Conditional survival of patients with metastatic renal-cell carcinoma treated with VEGF-targeted therapy: a population-based study. Lancet Oncol. 2012; 13: 927-35. doi:10.1016/S1470-2045(12)70285-1.

27. Escudier B. Emerging immunotherapies for renal cell carcinoma. Ann Oncol. 2012; 23 Suppl 8: viii35-40. doi:10.1093/annonc/mds261.

28. Hamid O, Robert C, Daud A, Hodi FS, Hwu WJ, Kefford R, et al. Safety and tumor responses with lambrolizumab (anti-PD-1) in melanoma. N Engl J Med. 2013; 369: 134-44. doi:10.1056/NEJMoa1305133.

29. Brahmer JR, Tykodi SS, Chow LQ, Hwu WJ, Topalian SL, Hwu P, et al. Safety and activity of anti-PD-L1 antibody in patients with advanced cancer. N Engl J Med. 2012; 366: 2455-65. doi:10.1056/NEJMoa1200694.

30. Bui MH, Seligson D, Han KR, Pantuck AJ, Dorey FJ, Huang Y, et al. Carbonic anhydrase IX is an independent predictor of survival in advanced renal clear cell carcinoma: implications for prognosis and therapy. Clin Cancer Res. 2003; 9: 802-11.

31. Cho D, Signoretti S, Dabora S, Regan M, Seeley A, Mariotti M, et al. Potential histologic and molecular predictors of response to temsirolimus in patients with advanced renal cell carcinoma. Clin Genitourin Cancer. 2007; 5: 379-85.

32. Vaziri SA, Tavares EJ, Golshayan AR, Rini BI, Aydin H, Zhou M, et al. Differing von hippel lindau genotype in paired primary and metastatic tumors in patients with clear cell renal cell carcinoma. Frontiers in oncology. 2012; 2: 51. doi:10.3389/fonc.2012.00051.

33. Gerlinger M, Rowan AJ, Horswell S, Larkin J, Endesfelder D, Gronroos E, et al. Intratumor heterogeneity and branched evolution revealed by multiregion sequencing. The New England journal of medicine. 2012; 366: 883-92. doi:10.1056/NEJMoa1113205.

34. Aziz SA, Sznol JA, Adeniran A, Parisi F, Kluger Y, Camp RL, et al. Expression of drug targets in primary and matched metastatic renal cell carcinoma tumors. BMC Clin Pathol. 2013; 13: 3. doi:10.1186/1472-6890-13-3.

35. Camp RL, Charette LA, Rimm DL. Validation of tissue microarray technology in breast carcinoma. Lab Invest. 2000; 80: 1943-9.

36. Brahmer JR, Drake CG, Wollner I, Powderly JD, Picus J, Sharfman WH, et al. Phase I study of single-agent anti-programmed death-1 (MDX-1106) in refractory solid tumors: safety, clinical activity, pharmacodynamics, and immunologic correlates. J Clin Oncol. 2010; 28: 3167-75. doi:JCO.2009.26.7609 [pii] 